\title{
ChemComm
}

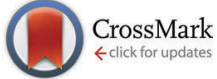

Cite this: Chem. Commun., 2015, 51, 7812

Received 19th February 2015 Accepted 2nd April 2015

DOI: $10.1039 / c 5 c c 01543 j$

www.rsc.org/chemcomm

\section{Rapid and simple preparation of remarkably stable binary nanoparticle planet-satellite assemblies $\dagger$}

\author{
Stefan Borsley, Sarah Flook and Euan R. Kay*
}

\begin{abstract}
We demonstrate a straightforward nonbiomolecular approach for self-assembly of binary NP planet-satellite superstructures, which display remarkable colloidal and structural stability under variations in temperature, $\mathrm{pH}$, ionic strength and solvent. The readily scalable process produces highly homogeneous samples and is tolerant to variations in building block size and shape, giving rapid access to a structurally diverse range of robust binary NP assemblies.
\end{abstract}

Building predictable three-dimensional (3D) structures from nanoparticle (NP) building blocks currently represents one of the foremost challenges in nanomaterials research. The interesting and often unique properties defined by NP chemical composition, size and shape, also crucially depend on assembly structure when several NPs are brought together. ${ }^{1}$ Although lithographic techniques can create small numbers of 2D nanostructures with exquisite precision, ${ }^{2}$ bottom-up wet chemical approaches have the potential to produce 3D NP assemblies in bulk quantities and with essentially no limits on feature size. ${ }^{1,3}$ Yet, current strategies for functionalization and assembly of colloidal NPs remain under-developed, and certainly fall far short of the precise control and mechanistic understanding achieved for the assembly of molecular building blocks.

The unique optoelectronic properties of noble metal NPs are significantly modified within NP assemblies, on account of strong near-field coupling between surface plasmons. ${ }^{2,3}$ These effects have been widely investigated, both from a fundamental perspective, and for a range of applications including colorimetric sensors, ${ }^{4}$ surface-enhanced spectroscopies, ${ }^{5}$ and nanometrology. ${ }^{6}$ Planet-satellite assemblies consisting of a single NP 'planet' surrounded by several NP 'satellites' are an attractive architecture in this context. Combining two different building blocks to achieve discrete assemblies of this sort can lead to a diverse

EaStCHEM School of Chemistry, University of St Andrews, North Haugh,

St Andrews KY16 9ST, UK. E-mail: ek28@st-andrews.ac.uk

$\dagger$ Electronic supplementary information (ESI) available: Full synthetic procedures and characterization for molecular species and component NPs; NMR, UV-vis, DLS and TEM data for component NPs and NP assemblies; results of control experiments and planet-satellite stability experiments. See DOI: $10.1039 / \mathrm{c} 5 \mathrm{cc} 01543 \mathrm{j}$ range of well-defined colloidally stable superstructures bearing a multitude of field enhancement 'hotspots' located within precisely controlled interparticle gaps. ${ }^{3 c}$ Previously, such structures have been achieved using oligonucleotide-directed selfassembly. ${ }^{7}$ However, this strategy is restricted to relatively large interparticle distances (hence weak plasmon coupling) and to a narrow window of environmental conditions. Alternatively, manipulation of solution ionic strength can lead to aggregation of charge-stabilized NPs, ${ }^{8}$ but this demands very careful control of conditions in order to achieve - and subsequently maintain a desired aggregate architecture. More recently, covalently linked strategies have produced some exciting results, yet it has proven challenging to produce uniform structures, ${ }^{9}$ without requiring preparation of complex macromolecular linkers, ${ }^{10}$ or undertaking multi-step assembly, stabilization and purification protocols. ${ }^{10,11}$

Here, we present an operationally simple and rapid noncovalent strategy for fabrication of binary planet-satellite assemblies in high yield and purity, from readily available AuNP building blocks. The assembly process takes place in aqueous solution, leading to the isotropic, 3D coverage of planet NPs of a variety of sizes and shapes, with smaller satellite NPs. The self-assemblies show remarkable stability to variations in temperature, solution $\mathrm{pH}$ and electrolyte content; they may also be isolated and stored as solids, then subsequently redispersed in either aqueous or organic solvents.

Water-soluble AuNP-1 (Fig. 1), stabilized by a homogeneous monolayer of 1,2-dihydroxy-terminated alkyl thiols, was prepared in a single step by a modification of the method developed by Stucky and co-workers, ${ }^{12}$ whereby $\mathrm{Au}\left(\mathrm{PPh}_{3}\right) \mathrm{Cl}$ is reduced with tertbutylamine borane complex (TBAB) in the presence of disulfide $\mathbf{1}_{2}$ (see ESI $\dagger$ for full experimental procedures). The nanoparticles were purified by precipitation and washing, and the absence of unbound molecular species was demonstrated by ${ }^{1} \mathrm{H}$ NMR spectroscopy (Fig. S1, ESI $\dagger$ ). Analysis by TEM (Fig. 2a and Fig. S2, $\mathrm{ESI} \dagger$ ) revealed spherical NPs with diameter $5.73 \pm 0.85 \mathrm{~nm}$.

Addition of an aqueous solution of AuNP-1 $\left(0.1 \mathrm{mg} \mathrm{mL}{ }^{-1}\right.$, $54 \mathrm{nM}$ ) to $40 \mathrm{~nm}$ citrate-stabilized AuNPs (0.1 nM) produced an 

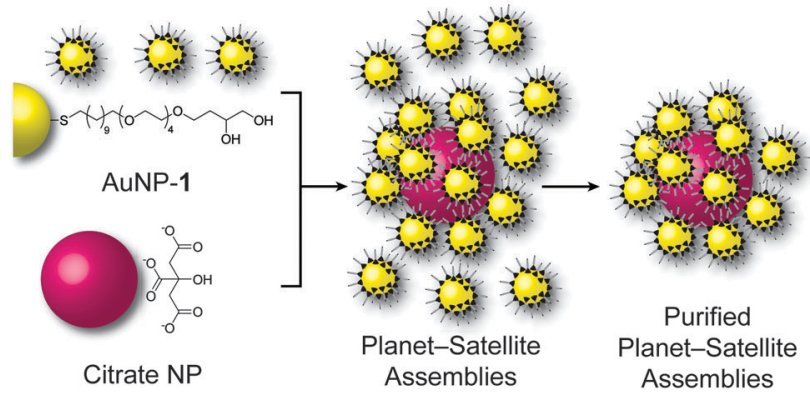

Fig. 1 Schematic representation of planet-satellite self-assembly. An aqueous solution of AuNP-1 is mixed with citrate-stabilized NPs, leading to rapid formation of planet-satellite structures, which may subsequently be purified by centrifugation and redispersion.

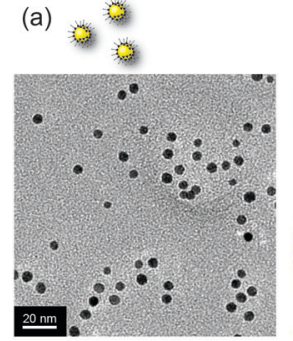

(c)
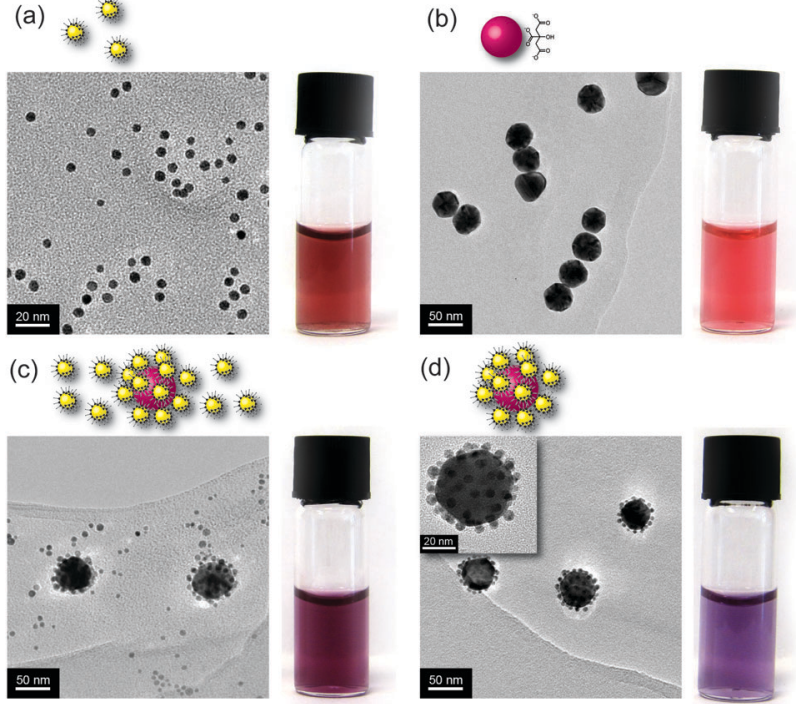

(d)
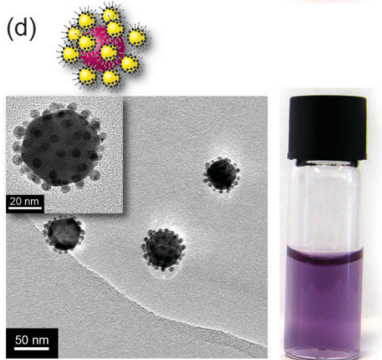

Fig. 2 TEM and visual characterization of component NPs and planetsatellite NP assemblies. (a) AuNP-1; (b) citrate-stabilized AuNPs (40 nm); (c) AuNP-1: citrate-NP (280:1); (d) purified planet-satellite assemblies. Photographs show the aqueous dispersions obtained at each stage.

immediate colour change from red to purple (compare Fig. 2a-c). UV-vis spectroscopy confirmed a red shift in the surface plasmon resonance (SPR) band relative to the component NPs, consistent with formation of NP aggregates (Fig. 3a). On increasing the stoichiometric ratio of AuNP-1 : citrate-NP, the SPR red shift was found to increase sharply, reaching a maximum shift at a ratio of $280: 1$, before exhibiting a gradual decrease in red shift at higher ratios (Fig. S17, ESI $\dagger$ ). We reasoned that the maximum shift in SPR wavelength corresponds to the optimum stoichiometric ratio for achieving isotropic planet-satellite assemblies, with incomplete planet NP coverage below this value and an increasing excess of unbound satellite NPs dominating the optical response at higher ratios. Without requiring any additional stabilizers, TEM images consistent with this interpretation were readily obtained. Images of the 280:1 mixture revealed that all large NPs were fully covered with a shell of smaller AuNP-1, together with a relatively small number of unbound AuNP-1 (Fig. 2c and Fig. S18, ESI $\dagger$ ).
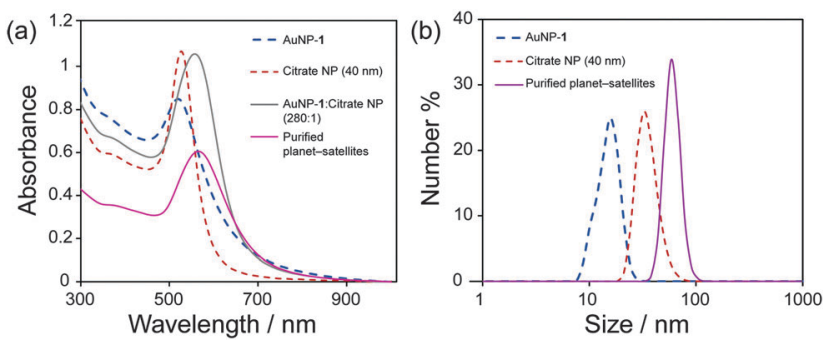

Fig. 3 (a) UV-vis spectra of component NPs and planet-satellite NP assemblies. (b) DLS measurements for component NPS and planet-satellite NP assemblies.

At higher ratios, the planet-satellite assemblies remained unchanged, simply accompanied by an increasing excess of isolated AuNP-1 on the grid. On the other hand, at ratios below $280: 1$, most citrate-NPs were associated with an incomplete shell of satellites, and isolated AuNP-1 were rarely observed (Fig. S18, ESI $\dagger$ ).

The observation of unbound AuNP-1, even at the optimized mixing ratio, prompted us to develop a purification procedure that would allow isolation of complete planet-satellite assemblies. A simple and rapid protocol was arrived at, involving centrifugation at $5900 \mathrm{rcf}$ for 4 minutes, leading to partial precipitation. The lightly coloured supernatant was decanted and the precipitate redispersed in the same volume of water. TEM imaging at this stage revealed that a small number of free AuNP-1 remained (Fig. S19, ESI†). The sample was therefore subjected to a second round of centrifugation at $2900 \mathrm{rcf}$ for 4 minutes. Following removal of the supernatant and redispersion of the precipitate, TEM at both low and high magnifications now revealed no persisting unbound AuNP-1, and the sample now consisted exclusively of planet-satellite superstructures (Fig. 2d and Fig. S19, ESI†). The purification process proved to be reproducible and scalable; routinely carried out on batches of up to $10 \mathrm{~mL}$, limited only by centrifuge tube size. TEM images of the supernatant solution (Fig. S20, ESI $\dagger$ ) showed only a small number of planet-satellite structures were removed along with the excess of unbound satellites, representing very small losses and, correspondingly, an efficient purification process.

Consistent with the removal of excess AuNP-1, purification resulted in a further red shift in SPR maximum wavelength to $564 \mathrm{~nm}$, a quite significant total shift of $\sim 40 \mathrm{~nm}$ from either of the isolated component NP building blocks (Fig. 3a). The strong plasmon coupling responsible for such a large shift results from the complete isotropic coverage of the large planet NPs with satellites held at short interparticle distances (Fig. 2d, inset). From TEM images of the purified samples, an average of 70 satellites surrounding every planet can be estimated (Fig. S21, ESI $\dagger$ ). With the purified samples in hand, the integrity of the assemblies in solution could be corroborated by dynamic light scattering (DLS) measurements, which revealed a monomodal distribution (Fig. 3b). The mean hydrodynamic diameter of $54 \mathrm{~nm}$ is in good agreement with the expected size of planetsatellite assemblies.

The planet-satellite NP assemblies exhibit remarkable structural and colloidal stability under a variety of conditions. No evidence of 

assemblies $\mathrm{NP}(40 \mathrm{~nm})$

(c)

AuNP-1 (a) Planet-satellite

(b) Citrate-stabilized

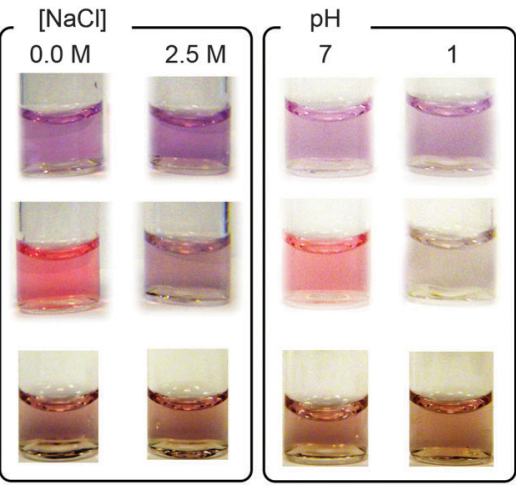

Fig. 4 Images of aqueous dispersions of planet-satellites (a), citratestabilized NPs (b) and AuNP-1 (c) demonstrating remarkable stability of the binary assemblies under conditions of increased ionic strength and decreased $\mathrm{pH}$.

disassembly or aggregation was observed either by eye or by UV-vis analysis upon heating the sample to $80^{\circ} \mathrm{C}$ for three hours (Fig. S24, $\mathrm{ESI} \dagger$ ). Similarly, the assemblies remained stable in high ionic strength solutions, showing no change in the surface plasmon resonance upon addition of either $\mathrm{NaCl}$ or $\mathrm{MgCl}_{2}$ (up to $3 \mathrm{M}$ ), in-line with the behaviour of AuNP-1 alone. By comparison, the citrate-stabilized planet NPs aggregate at electrolyte concentrations $\leq 50 \mathrm{mM}$ (Fig. 4 and Fig. S28-S30, ESI $\dagger$ ). The planet-satellite assemblies are also stable under a wide range of $\mathrm{pH}$ values. Again mirroring the behaviour of AuNP-1, no aggregation, precipitation or disassembly was observed over the range $1 \leq \mathrm{pH} \leq 14$, in stark contrast to the precursor citrate-stabilized NPs, which readily precipitate under moderately acidic conditions (Fig. 4 and Fig. S31, ESI $\dagger$ ). It appears that the NP building blocks are strongly associated and that the robust colloidal stability of AuNP-1 is conferred on the entire assembly, without the need for any additional stabilizing or capping agents.

The facile purification process involving precipitation and redispersion suggested that the assemblies might be isolable as a solid for storage prior to redispersion in a range of suitable solvents. Aqueous solutions of purified planet-satellites were centrifuged to induce precipitation. Removal of the colourless supernatant left a film-like residue, which could subsequently be redispersed in water with no loss of material apparent by UV-vis analysis (Fig. S25a, ESI $\dagger$ ). Redispersion could also be achieved after drying in vacuo, although in this case a slight decrease in absorbance was observed (Fig. S25b, ESI $\dagger$ ), attributable to irreversible adsorption to the walls of the glass vial. However, no change in the shape or position of the SPR band was apparent, indicating the fidelity of the self-assembled structure. By contrast, solvent evaporation from a dispersion of citrate-stabilized NPs leads to irreversible aggregation, rendering redispersion impossible. The precipitation-redispersion protocol also allowed transfer from aqueous to organic environments; the planet-satellites were readily redispersible in $\mathrm{MeCN}$, with no change to the assembly structure apparent by either UV-vis or TEM imaging (Fig. S26 and S27, ESI $\dagger$ ).

Having established a protocol for assembly of these robust binary NP superstructures, we next sought to explore the generality (a)

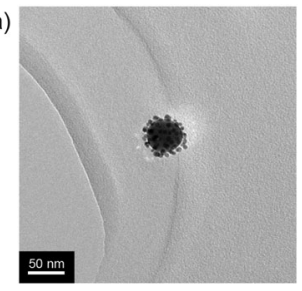

(c)

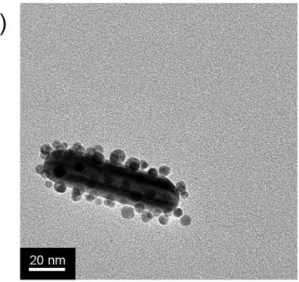

(b)

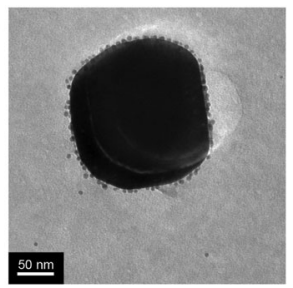

(d)

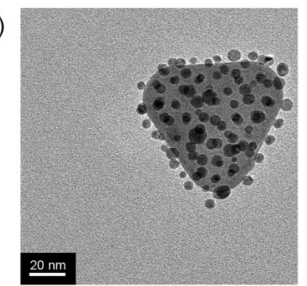

Fig. 5 Planet-satellite NP structures obtained by self-assembly of AuNP-1 and (a) spherical $30 \mathrm{~nm}$ diameter or (b) spherical $150 \mathrm{~nm}$ diameter citrate-stabilized NPs; and with (c) rod-shaped or (d) prismatic (d) citrate-stabilized NPs.

of this approach for different planet NPs. With minimal modification to the protocol, planet-satellite assemblies were successfully obtained from spherical citrate-stabilized NPs spanning a remarkably wide size range (and from both home-made or commercial sources, Fig. 5a and b and Fig. S32, ESI $\dagger$ ). The ability to assemble NP building blocks with different shapes substantially increases the range and complexity of possible structures and opportunities for property tuning. ${ }^{7 i}$ We therefore prepared a sample of citratestabilized NPs with a variety of sizes and shapes, including large numbers of rods and prisms. Planet-satellite assembly around non-spherical NPs proceeded efficiently, and purification from unbound AuNP-1 was readily achieved in the same manner as before (Fig. 5c and d and Fig. S33, ESI $\dagger$ ). As a preliminary attempt to expand the range of satellite NPs, we prepared $10 \mathrm{~nm}$ NPs stabilized with the dihydroxy-terminated ligand 1 via ligand exchange from citrate-stabilized NPs. On combination with $40 \mathrm{~nm}$ citrate-stabilized NPs, assembly was again observed. However, in this case the process was significantly slower and failed to produce well-defined planet-satellite structures (see Fig. S34, ESI $\dagger$ ). Most likely, repulsive electrostatic interactions resulting from residual citrate species on the satellite NPs create a more complex set of forces governing assembly and stability. We are currently investigating alternative methods for expanding the scope of satellite NPs.

The self-assembly process appears to be governed by a highly specific interaction between the 1,2-dihydroxy functionality on AuNP-1 and citrate-stabilized NPs of virtually any sort. On replacing the dihydroxy-functionalized NPs with monohydroxyfunctionalized AuNP-2 (Fig. S22, ESI $\dagger$ ), no NP aggregation was observed, either in solution (UV-vis and DLS) or by TEM (Fig. S23, ESI $\dagger$ ). It has recently been proposed that citrate binds to AuNP surfaces through two carboxylate-Au interactions, with the nonsurface-bound carboxylate involved in hydrogen bonding to a weakly associated citric acid adlayer. ${ }^{13}$ Despite the inherently limited stabilization provided by hydroxyl-based hydrogen bonds in aqueous environments, ${ }^{14}$ a cooperative multi-point interaction between the 1,2-dihydroxy motif on AuNP-1 and a NP-bound 
carboxylate may be sufficient to displace the secondary citric acid layer; the cumulative multivalent effect of several such interactions between the two densely functionalized NP surfaces could then explain the robust planet-satellite NP association. Aside from biomolecule-based systems, ${ }^{1 a, 3 b}$ multivalent hydrogen bonding under aqueous conditions has previously been invoked in only a handful cases to explain NP aggregation. ${ }^{15}$ Each of these involves only a single NP building block and achieves limited structural control and stability. In the current case, an alternative explanation could involve direct interaction between the 1,2-diols and the Au surface. Studies under ultrahigh vacuum conditions suggest that alcohols can adsorb weakly on noble metal surfaces at low temperatures, ${ }^{16}$ but it is clear from the results with monohydroxy-functionalized AuNP-2 that such a process alone is not sufficient to achieve NP self-assembly. A chelate interaction between the diols of AuNP-1 and metal surface atoms may of course enhance this interaction, yet would still require penetration and displacement of the surface-bound citrate monolayer and a significant Au-diol binding energy. Efforts are currently under way to elucidate the molecular details of the interactions involved.

In conclusion, we have developed a versatile and operationally simple protocol for the self-assembly and purification of 3D binary NP planet-satellite structures. The rapid, one-step assembly procedure does not require careful control of environmental conditions, eliminates the need for complex biological, supramolecular or macromolecular NP ligands, and is readily scalable. Isotropic coverage of the planet NPs can be achieved, irrespective of surface area, through optimization of building block stoichiometry using simple spectroscopic measurements. The highly stable characteristics of the satellite NPs are conferred on the binary assemblies, which are dispersible in either aqueous or organic media. This allows rapid and efficient purification via standard techniques, without recourse to additional capping or stabilizing steps. Short interparticle distances produce large SPR red shifts for the resulting planet-satellite assemblies, suggesting intense electromagnetic field enhancements at hotspots between the neighbouring NP surfaces. The level of control achieved here is highly encouraging in the quest to develop a generalizable NP building block toolkit that can rival the pre-eminent oligonucleotide approaches by exploiting the stability and structural diversity of nonbiomolecular synthetic chemistry.

This work was supported by the EPSRC (EP/K016342/1 and DTG), the University of St Andrews, and by a Royal Society of Edinburgh/Scottish Government Fellowship (E.R.K.). We are grateful to Prof. Garry Taylor and Dr Jane Potter for access to DLS instrumentation, and to Mr Ross Blackley for assistance with TEM measurements. Mass spectrometry was carried out at the EPSRC UK National Mass Spectrometry Facility (NMSF) at Swansea University.

\section{Notes and references}

1 (a) C. L. Choi and A. P. Alivisatos, Annu. Rev. Phys. Chem., 2010, 61, 369; (b) Z. H. Nie, A. Petukhova and E. Kumacheva, Nat. Nanotechnol., 2010, $5,15$.

2 N. J. Halas, S. Lal, W. S. Chang, S. Link and P. Nordlander, Chem. Rev., 2011, 111, 3913.

3 (a) S. K. Ghosh and T. Pal, Chem. Rev., 2007, 107, 4797; (b) S. J. Tan, M. J. Campolongo, D. Luo and W. Cheng, Nat. Nanotechnol., 2011, 6, 268; (c) J. M. Romo-Herrera, R. A. Alvarez-Puebla and L. M. LizMarzán, Nanoscale, 2011, 3, 1304.

4 (a) R. Elghanian, J. J. Storhoff, R. C. Mucic, R. L. Letsinger and C. A. Mirkin, Science, 1997, 277, 1078; (b) N. L. Rosi and C. A. Mirkin, Chem. Rev., 2005, 105, 1547.

5 (a) S. M. Nie and S. R. Emory, Science, 1997, 275, 1102; (b) K. Kneipp, Y. Wang, H. Kneipp, L. T. Perelman, I. Itzkan, R. Dasari and M. S. Feld, Phys. Rev. Lett., 1997, 78, 1667; (c) L. Guerrini and D. Graham, Chem. Soc. Rev., 2012, 41, 7085.

6 C. Sönnichsen, B. M. Reinhard, J. Liphardt and A. P. Alivisatos, Nat. Biotechnol., 2005, 23, 741.

7 (a) R. C. Mucic, J. J. Storhoff, C. A. Mirkin and R. L. Letsinger, J. Am. Chem. Soc., 1998, 120, 12674; (b) F. W. Huo, A. K. R. Lytton-Jean and C. A. Mirkin, Adv. Mater., 2006, 18, 2304; (c) X. Y. Xu, N. L. Rosi, Y. H. Wang, F. W. Huo and C. A. Mirkin, J. Am. Chem. Soc., 2006, 128, 9286; (d) H. Yao, C. Q. Yi, C.-H. Tzang, J. J. Zhu and M. S. Yang, Nanotechnology, 2007, 18, 015102; (e) D. S. Sebba, J. J. Mock, D. R. Smith, T. H. LaBean and A. A. Lazarides, Nano Lett., 2008, 8, 1803; $(f)$ M. M. Maye, D. Nykypanchuk, M. Cuisinier, D. van der Lelie and O. Gang, Nat. Mater., 2009, 8, 388; $(g)$ S.-Y. Chen and A. A. Lazarides, J. Phys. Chem. C, 2009, 113, 12167; $(h)$ S. Pal, J. Sharma, H. Yan and Y. Liu, Chem. Commun., 2009, 6059; (i) L. G. Xu, H. Kuang, C. L. Xu, W. Ma, L. B. Wang and N. A. Kotov, J. Am. Chem. Soc., 2012, 134, 1699; $(j)$ L. H. Tan, H. Xing, H. Chen and Y. Lu, J. Am. Chem. Soc., 2013, 135, 17675; (k) L. Y. T. Chou, K. Zagorovsky and W. C. W. Chan, Nat. Nanotechnol., 2014, 9, 148.

8 N. Gandra, A. Abbas, L. Tian and S. Singamaneni, Nano Lett., 2012, 12, 2645.

9 N. Gandra and S. Singamaneni, Chem. Commun., 2012, 48, 11540. 10 C. Rossner and P. Vana, Angew. Chem., Int. Ed., 2014, 53, 12639.

11 (a) Y. Wang, G. Chen, M. Yang, G. Silber, S. Xing, L. H. Tan, F. Wang, Y. Feng, X. Liu, S. Li and H. Chen, Nat. Commun., 2010, 1, 87; (b) J. H. Yoon, J. Lim and S. Yoon, ACS Nano, 2012, 6, 7199.

12 N. Zheng, J. Fan and G. D. Stucky, J. Am. Chem. Soc., 2006, 128, 6550. 13 J.-W. Park and J. S. Shumaker-Parry, J. Am. Chem. Soc., 2014, 136, 1907.

14 C. A. Hunter, Angew. Chem., Int. Ed., 2004, 43, 5310.

15 (a) J. Simard, C. Briggs, A. K. Boal and V. M. Rotello, Chem. Commun., 2000, 1943; (b) K. G. Thomas, S. Barazzouk, B. I. Ipe, S. T. S. Joseph and P. V. Kamat, J. Phys. Chem. B, 2004, 108, 13066; (c) Z. Sun, W. Ni, Z. Yang, X. Kou, L. Li and J. Wang, Small, 2008, 4, 1287; (d) I. I. S. Lim, D. Mott, W. Ip, P. N. Njoki, Y. Pan, S. Q. Zhou and C. J. Zhong, Langmuir, 2008, 24, 8857; (e) N. Varghese, S. R. C. Vivekchand, A. Govindaraj and C. N. R. Rao, Chem. Phys. Lett., 2008, 450, 340; $(f)$ W. H. Ni, R. A. Mosquera, J. Perez-Juste and L. M. Liz-Marzán, J. Phys. Chem. Lett., 2010, 1, 1181.

16 (a) B. Xu, X. Liu, J. Haubrich, R. J. Madix and C. M. Friend, Angew. Chem., Int. Ed., 2009, 48, 4206; (b) X. Liu, B. Xu, J. Haubrich, R. J. Madix and C. M. Friend, J. Am. Chem. Soc., 2009, 131, 5757. 